\title{
Non-invasive femoropopliteal assessment: is that angiogram really necessary?
}

\author{
C P SHEARMAN, B R GWYNN, F CURRAN, M X GANNON, M H SIMMS
}

\begin{abstract}
A method of non-invasive preoperative assessment of chronically ischaemic legs was developed that used clinical data and data derived from Doppler ultrasonography to produce a numerical score that could be compared with an angiographic score for stenosis of the popliteal artery trifurcation. The two scoring systems were applied retrospectively to 144 legs after femorodistal bypass. A close correlation was observed $(r=0.89$, $\mathbf{p}<\mathbf{0 . 0 0 1}$ ), and both systems tended to predict the level of grafting undertaken. A prospective comparison was then made in 81 ischaemic legs that were examined by arteriography; the correlation between the two scoring systems remained close $(r=0.89$, $p<0.001$, and the level of bypass was correctly predicted by the non-invasive assessment in $\mathbf{4 4}$ of 50 legs that were operated on. Use of the non-invasive assessment subsequently greatly reduced the indications for preoperative arteriography in patients requiring femorodistal vascular reconstruction.
\end{abstract}

\section{Introduction}

Over the past decade vascular surgeons have become increasingly willing to attempt reconstructive rather than ablative procedures in patients with severe ischaemia of the legs. ${ }^{\prime}$ This trend has highlighted the shortcomings of conventional preoperative arteriography in showing detailed arterial anatomy in legs with reduced blood flow, when roughly a quarter of crural or pedal arteries that are judged patent on Doppler ultrasonography or direct operative exploration may fail to opacify. ${ }^{2-4}$ Though the use of reactive hyperaemia and other specialised radiological techniques may enhance visualisation of distal vessels in skilled hands, an increasing number of surgeons now plan their vascular reconstructions on the basis of intraoperative arteriography before bypass, sometimes supplemented by Doppler insonation. ${ }^{25}$

In patients with an adequate femoral pulse and disease of the femoropopliteal segment the decision to bypass to the popliteal trunk or directly on to calf vessels depends on the extent of occlusion at the popliteal trifurcation, which influences flow and patency in femoropopliteal grafts. ${ }^{67}$ Bypass to isolated popliteal segments has

Department of Surgery, Selly Oak Hospital, Birmingham B29 6JH

C P SHEARMAN, FRCS, surgical registrar

B R GWYNN, FRCS, research registrar

F CURRAN, FRCS, surgical registrar

M H SIMMS, FRCS, consultant (and at Queen Elizabeth Hospital)

Queen Elizabeth Hospital, Birmingham

M X GANNON, FRCS, research registrar

Correspondence to: $\mathrm{Mr}$ Simms. its proponents, ${ }^{89}$ but restoration of a normal ankle arterial pressure is seldom achieved by this operation ${ }^{10}$ and limb salvage rates are inferior to those that can be achieved with bypass to crural arteries. ${ }^{11}$ Our experience suggests that patients with severe stenosis of the popliteal artery trifurcation are better served with a direct crural bypass. ${ }^{12}$

Operative arteriography usually shows the popliteal and crural arteries sufficiently to indicate the correct level of bypass. It might be argued, however, that it is preferable to make this decision preoperatively; operating time and facilities can then be reserved appropriately and patients given a more accurate estimate of the prospects of success. Unfortunately, not only may preoperative arteriograms misrepresent patency in vessels distal to the proposed site of the graft but they cannot easily be repeated to take account of sudden deteriorations in perfusion of the limb. Consequently, a reliable and repeatable non-invasive means of assessing the condition of the popliteal artery trifurcation and vessels distal to the proposed graft would be of practical clinical value. Although clinical information and isolated physiological measurements such as ankle systolic arterial pressure,${ }^{13}$ transcutaneous oxygen tension, ${ }^{14}$ and skin blood flow ${ }^{15}$ have been used to grade the severity of ischaemia of the legs, such data alone do not indicate the anatomy of underlying arterial disease and give little or no indication of the prospects for vascular reconstruction.

We devised a non-invasive scoring system for ischaemic legs that combines clinical data with Doppler ultrasonographic assessment of the patency and pressure of the ankle arteries. This score was designed to be compatible with a simple angiographic scoring system for the popliteal trifurcation. We then compared the two scoring systems retrospectively and prospectively in patients undergoing vascular assessment and reconstruction to ascertain whether non-invasive scoring could predict arteriographic findings.

\section{Methods and patients}

\section{ARTERIOGRAPHIC SCORE}

The anterior tibial, posterior tibial, and peroneal arteries were assessed for the first $5 \mathrm{~cm}$ from their popliteal origin as normal (2 points), diseased with $50 \%$ or more stenosis (1), or occluded (0), giving a possible range from 0 to 6 (fig 1).

\section{NON-INVASIVE SCORE}

The non-invasive score was obtained by summing the points obtained from clinical studies and studies of the patency and pressure of the ankle artery as follows.

Clinical data-Three points were awarded initially, then 1 point was deducted if the patient's claudication distance was under $50 \mathrm{~m}, 2$ points if the patient currently had rest pain, and all three points if there was ischaemic or trophic change (ulceration, fissuring, or gangrene).

Patency-The anterior tibial, posterior tibial, and peroneal arteries 
(usually, in practice, the anterior communicating branch) were insonated at ankle level by means of an $8 \mathrm{MHz}$ Doppler probe with the patients supine and rested. For each patent artery (defined as one giving a pulsatile signal) 0.5 points was awarded, giving a maximum of 1.5 points

Pressure-An $8 \mathrm{MHz}$ Doppler ultrasound probe and a standard sphygmomanometer cuff around the distal calf were used with the patient supine to determine systolic pressure in each of the three ankle vessels. The highest of these values was then divided by the brachial systolic pressure to determine the ankle:brachial pressure index and this then multiplied by three to give the pressure score.

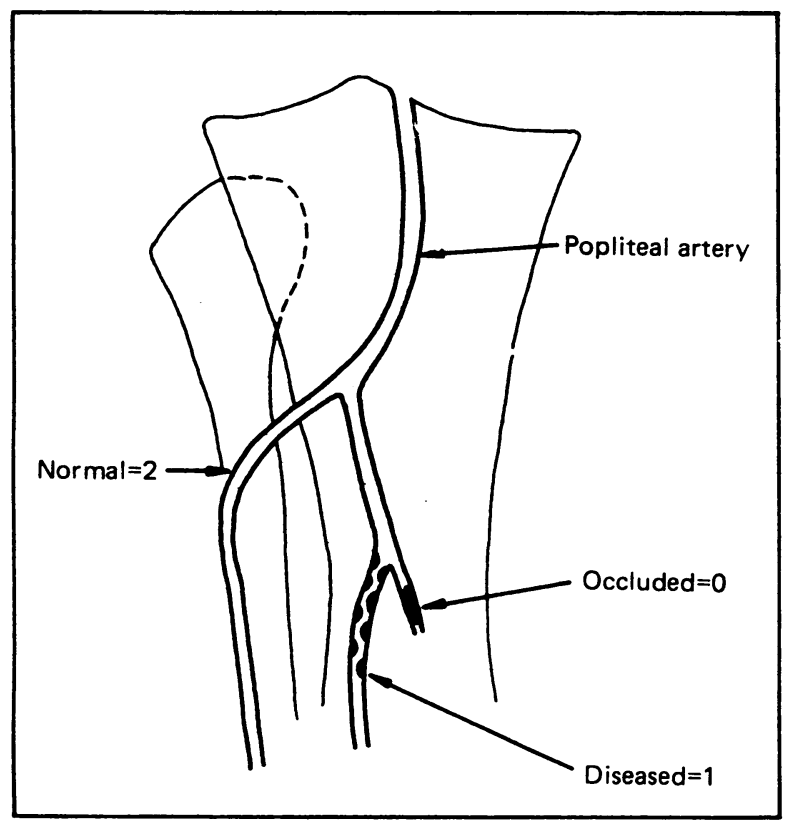

FIG 1-For the angiographic score the first $5 \mathrm{~cm}$ from its origin of each of the main crural vessels is scored as 0,1 , or 2 , depending on patency, giving a total of $0-6$ points.

To determine which crural artery was most suitable for bypass we used the pedal arch patency test of Roedersheimer $e t a l$, in which the deep plantar artery in the first interdigital cleft is insonated while each of the three crural arteries in turn is occluded at ankle level with a finger.$^{16}$ If necessary this can be done with the leg dependent to augment the Doppler signal.

With the above method complete assessment of each ischaemic limb can be performed in about five minutes.

\section{PATIENTS}

We calculated the non-invasive and angiographic scores retrospectively in 144 ischaemic legs that had been examined by angiography and then operated on by femorodistal reconstruction and for which clinical and Doppler ultrasonographic data had been recorded. Arteriograms were scored by two people who did not know the non-invasive scores, and vice versa. We then recorded non-invasive scores for one or both legs in a separate series of patients who were about to undergo arteriography and vascular reconstruction. We noted our prediction of the likely site of the distal end of the graft based on a combination of the non-invasive score and the results of insonation of the pedal arch. Angiographic information was available on 81 legs, of which 50 subsequently were operated on by vascular reconstruction (most of those not operated on were the contralateral leg of patients undergoing unilateral vascular reconstruction). The site for the distal anastomosis of the graft was selected on the basis of the arteriograms and the findings at exploration. In 19 of the legs that were to be operated on the femoral pulse was judged clinically to be absent or sufficiently weak to necessitate an inflow procedure (aortofemoral, axillofemoral, or cross femoral). The non-invasive scores in these legs were corrected for inflow disease by the addition of two points for an absent femoral pulse and one for a weak pulse. The decision to perform an inflow procedure in these patients was based on preoperative palpation of the femoral artery, which is as accurate as any instrumental technique in detecting appreciable $(>50 \%)$ aortoiliac stenosis. ${ }^{17}$

\section{Results}

\section{RETROSPECTIVE SERIES}

We scored 144 legs that had been operated on by femoropopliteal (42) or femorocrural (102) bypass. There was good correlation between the angiographic and non-invasive assessment $(r=0.89, p<0.001$; fig 2), although the absolute values for the non-invasive assessment tended to be 1 point greater than the corresponding angiographic scores throughout. The

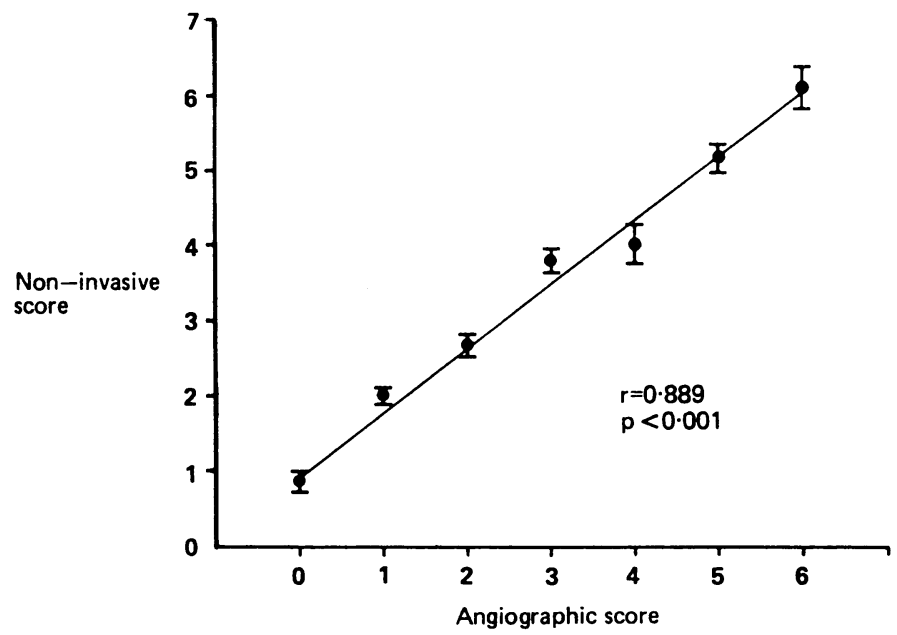

FIG 2-Correlation between non-invasive scores and angiographic scores in 144 legs operated on by femorodistal bypass, studied retrospectively. Points are means (SEM)

non-invasive scores accorded with the level of bypass, patients scoring more than 3 requiring femoropopliteal bypass (fig 3). Only two of the 42 femoropopliteal grafts $(48 \%)$ and eight of the 102 femorocrural grafts $(8 \%)$ failed to fit this separation.

\section{PROSPECTIVE SERIES}

Eighty eight legs were examined non-invasively and by arteriography either preoperatively (61) or intraoperatively (27). Seven arteriograms failed to show the popliteal trifurcation sufficiently to allow scoring, and thus we compared the non-invasive and angiographic scores in 81 legs. Once again a close correlation was obtained $(r=0.89, p<0.001$; fig 4$)$. The correlation was maintained in the 19 legs for which an inflow correction factor was required $(r=0.91)$.

Reconstruction was performed on 50 legs, because of severe claudication (two) and rest pain or gangrene (48). Three levels of bypass were used, the graft extending to the popliteal trunk, to the popliteal trifurcation (using a medial below knee popliteal approach and detaching the soleus arch as necessary), or, in 19 legs, directly to one or two arteries in the mid- or distal calf. Forty eight grafts were of autogenous vein, usually the long saphenous vein in situ, and two were of $6 \mathrm{~mm}$ polytetrafluoroethylene. Thrombosis occurred in five grafts perioperatively (two popliteal and three crural), in each case because of technical problems related to the graft: two legs had to be amputated; in one an inflow procedure remained patent, providing adequate distal perfusion; claudication continued to occur in one as before; and in the fifth slow healing of ischaemic ulceration occurred, presumably due to a concomitant profundaplasty. Two further legs were amputated despite functioning grafts, one because of secondary graft haemorrhage through an infected wound and the other because of progressive painful gangrene in the foot, probably due to a technically inadequate anastomosis at the trifurcation. Although this graft was revised and extended distally, irreversible ischaemic damage had already occurred.

As in the retrospective series, non-invasive score tended to predict the level of bypass performed. Taking scores of $0-2$ as predicting femorocrural bypass, $>2-<4$ as predicting femorotrifurcation bypass, and $\geqslant 4$ as predicting bypass to the popliteal trunk, two popliteal and four crural bypasses were performed when non-invasive assessment had suggested that trifurcation bypass was required. Thus there was agreement between the non-invasive prediction and surgical outcome in $88 \%$ of cases. Furthermore, 
there was a highly significant difference between the mean non-invasive scores for the three levels of bypass (femoropopliteal $v$ femorotrifurcation $\mathrm{p}<0.001$, femorotrifurcation $v$ femorocrural $\mathrm{p}<0.001$; Student's $t$ test).

\section{Discussion}

An ideal system of assessing ischaemia of the legs would be noninvasive, safe, simple, and rapid to perform. It would provide both a global index of the severity of disease and sufficient information on arterial patency for a reconstructive procedure to be planned. It could therefore be repeated as often as desired to monitor progression of disease until immediately before surgery. Our experience of
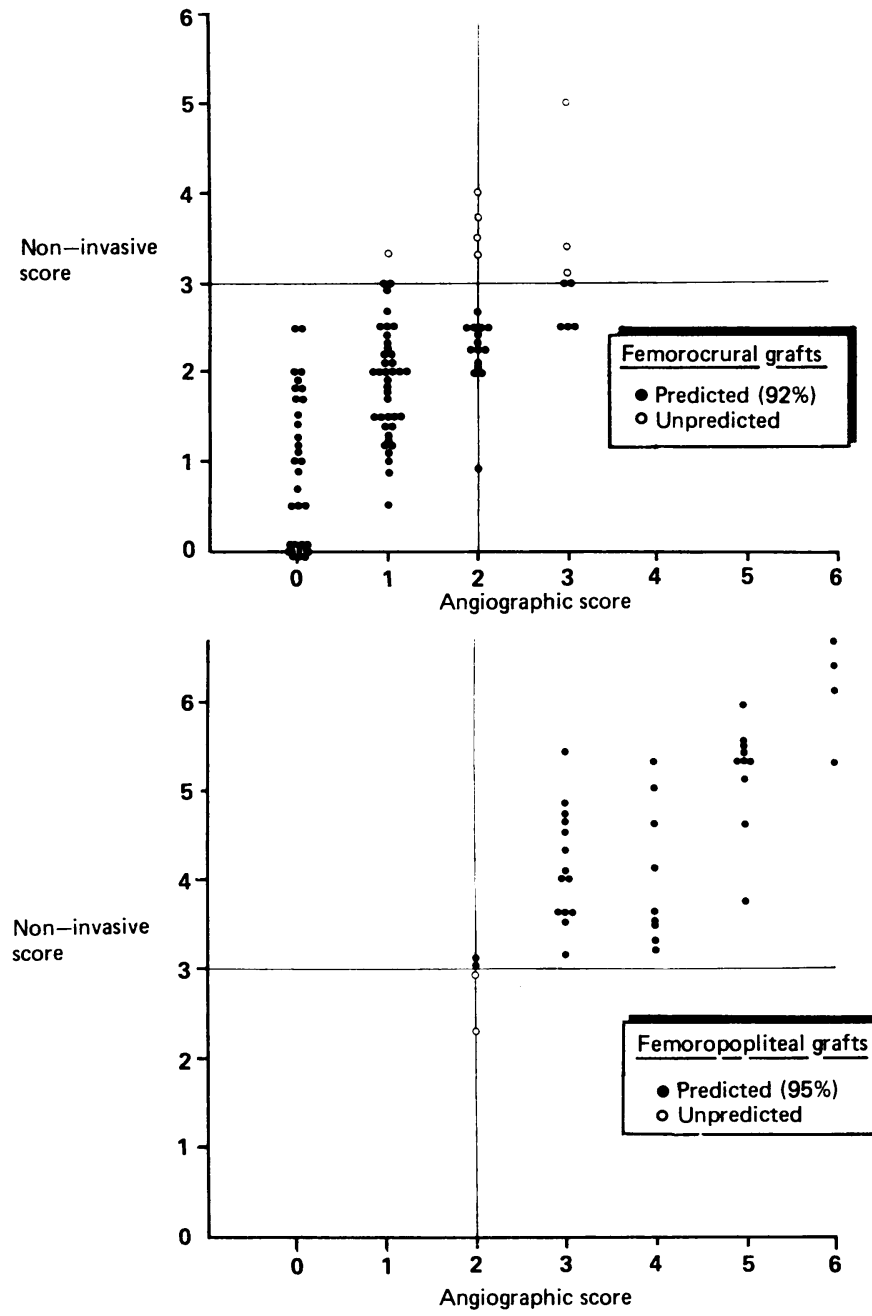

FIG 3-Retrospective non-invasive and angiographic scores for 144 legs treated by femorodistal grafting. Those with non-invasive scores of $\leqslant 3$ or angiographic scores of $\leqslant 2$ tended to be treated by femorocrural bypass (top); others tended to be treated by femoropopliteal bypass (bottom).

non-invasive scoring suggests that it conforms surprisingly well to this theoretical ideal. The simplicity, safety, and speed of the method are self evident, and it may be performed whenever or wherever desired.

Non-invasive scoring does not seem to be particularly variable or observer dependent. All 50 legs in our prospective series were assessed independently by two observers on separate occasions, and discrepancies were recorded in only five legs, the maximum discrepancy being 0.8 point. (Similarly, we found a difference of one point in less than $10 \%$ of over 200 arteriograms scored by separate observers.)

Since we adopted this non-invasive method of assessment our

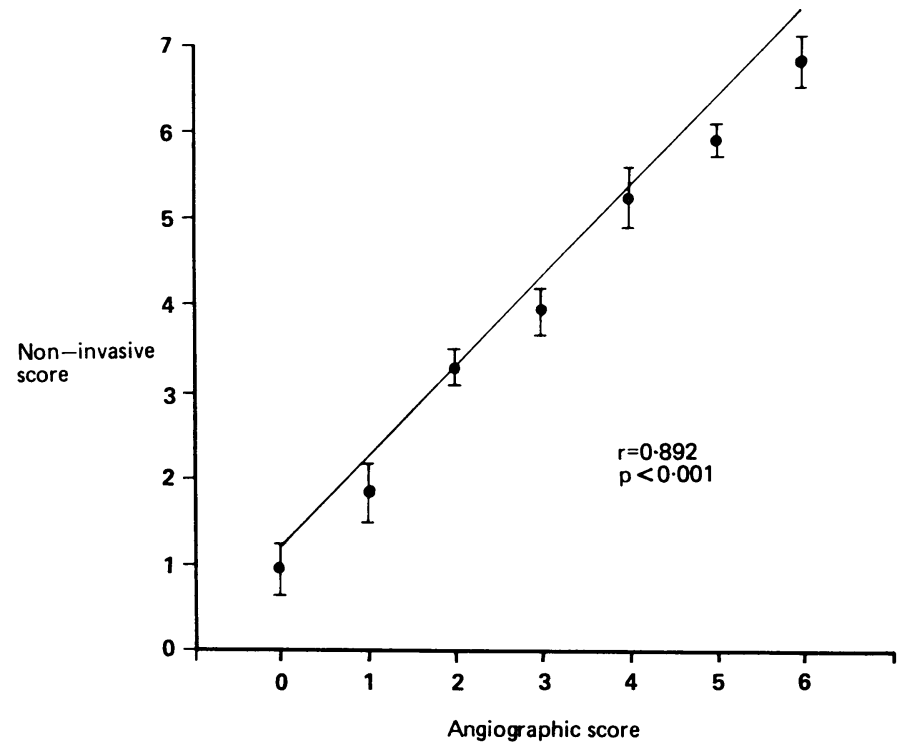

FIG 4-Correlation between non-invasive and angiographic scores determined prospectively in 81 limbs examined by arteriography before vascular reconstruction. Points are means (SEM).

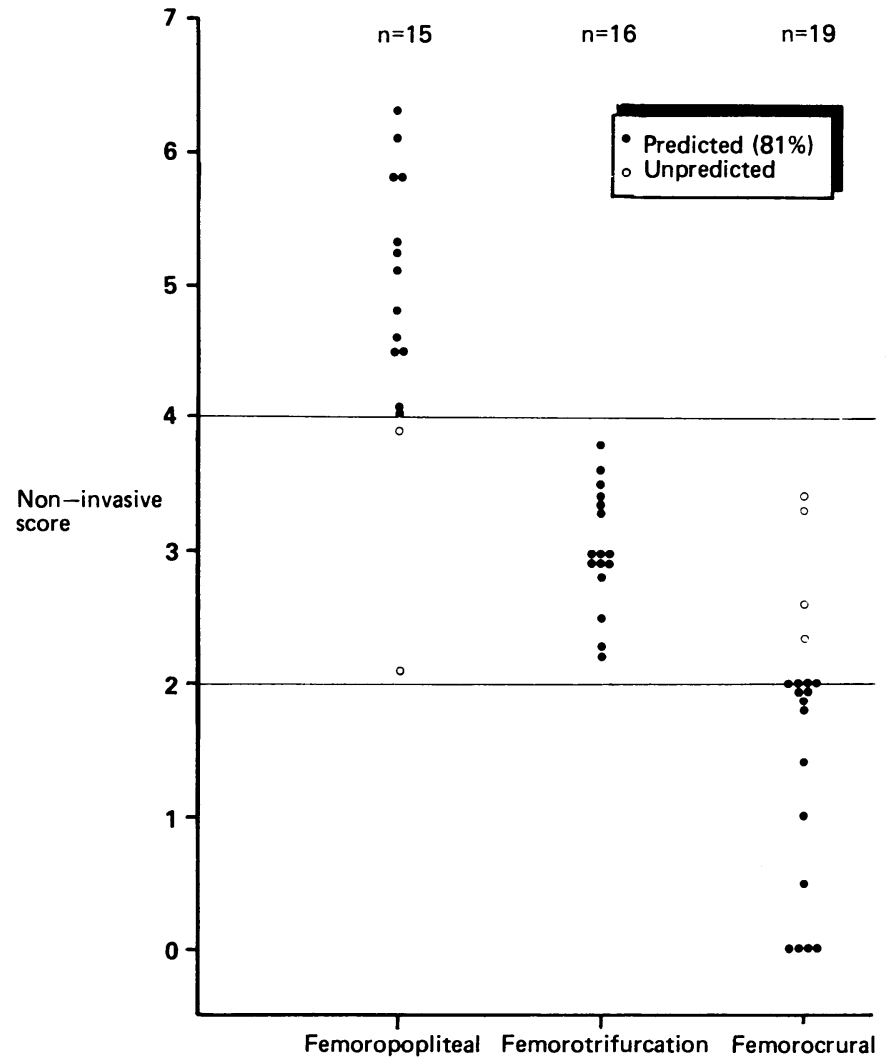

FIG 5-Results of prospective study, showing tendency for non-invasive scores to predict level of bypass required: $0-2$, crural; $>2-<4$, trifurcation; $\geqslant 4$, popliteal trunk.

use of preoperative arteriography has fallen drastically. Of 36 femorodistal procedures performed over the past six months, only seven were preceded by preoperative arteriography (usually to define aortoiliac disease). In practice, after the non-invasive score has been obtained to indicate operative strategy two surgeons work synchronously to prepare inflow and outflow sites. Usually an intraoperative arteriogram is obtained before bypass, ${ }^{5}$ and this takes only a few minutes. This serves as a "fail safe" precaution and as a standard against which to judge the accuracy of the non-invasive 
score. Our virtual abandonment of preoperative arteriography has brought savings in time, money, and patient discomfort, while our limb salvage rate has remained steady at over $90 \%$. Though non-invasive scoring cannot provide an anatomical picture, in conjunction with insonation of the pedal arch it does seem to indicate the correct site for distal anastomosis of the graft, and in practice this is all that is required. Provided that the surgical team is prepared to undertake bypass to all levels in the leg, non-invasive scoring can be the sole preoperative strategic investigation in most patients needing femorodistal bypass. We consider that the method merits wider assessment.

We thank Professor F Ashton and Professor Sir Geoffrey Slaney for allowing us to study their patients. We are also grateful to Christine Hail and Jane Randell for the photography, and to Moira Owen for typing the manuscript.

\section{References}

1 Bell PRF. Are distal vascular procedures worthwhile? Br $\mathcal{F}$ Surg 1985;72:335

2 Ricco JB, Pearce WH, Yao JST, Flynn W'R, Bergan JJ. The use of operative pre-bypass arteriography with Doppler ultrasound recordings to select patients for extended femoro-distal bypass. Ann Surg 1983;198:646-53
3 Simms MH, Hardman J, Slaney G. The relevance of pre-bypass pedal arch patency assessment. $B r$ I Surg 1984;71:381.

Campbell WB, Fletcher EL, Hands LJ. Assessment of the distal lower limb arteries: a comparison of arteriography and Doppler ultrasound. Ann R Coll Surg Engl 1986;68:37-9.

5 Flannigan DP, Williams LR, Keifer T, Schular JJ, Behrend AJ. Pre-bypass operative arteriography. Surgerv 1982;92:627-33.

6 Terry $\mathrm{HJ}$, Allan JS, Taylor GW. The relationship between blood flow and failure of femoropopliteal arterial occlusion. $\mathrm{Br} \mathcal{F}$ Surg 1972;59:549-51.

7 Grimley RP, Obeid ML, Ashton F, Slaney G. Long term results of autogenous vein bypass grafts in femoro-popliteal arterial occlusion. Br f Surg 1979;66:723-6.

8 Corson JD, Brewster DC, Lasalle AJ, Darling RC. Comparative analysis of veins and prosthetic bypass grafts to the isolated popliteal artery. Surgery 1982;91:448-51.

9 Kaufman JL, Whitemore AD, Couch NP, Mannick JA. The fate of bypass grafts to an isolated popliteal artery segment. Surgery 1982;92:1027-31.

10 Mason R, Lanfranchi A, Giron F. Isolated popliteal versus distal bypasses for limb salvage. Surg Gynecol Obstet 1982;155:49-53.

11 Leather RP, Shah DM, Karmody DM. Infra-popliteal arterial bypass for limb salvage: increased patency and utilisation of the saphenous vein used "in situ." Surgery 1981;90:1000-8.

12 Gannon MX, Simms MH, Ruddock S, Goldman M, Ashton F, Slaney G. Peri-operative complication of in-situ saphenous vein grafts. Ann $R$ Coll Surg Engl 1986;68:134-6.

13 Bell PRF, Charlesworth D, De Palma RH, et al. The definition of critical ischaemia of a limb. $\mathrm{Br} \mathcal{F}$ Surg 1982;69(suppl):S2.

14 Spence VA, McCollum PT. Evaluation of the ischaemic limb by transcutaneous oxymetry. In: Greenhalgh RM, ed. Diagnostic vechniques and assessment procedures in vascular surgery. London: Grune and Stratton, 1985.331-41.

15 Lassen NA, Holstein P. Uses of radioisotopes in assessment of distal blood flow and distal blood pressure in arterial insufficiency. Surg Clin N Am 1974;54:39-55.

16 Roedersheimer LR, Feins R, Green RM. Doppler evaluation of the pedal arch. Ann 7 Surg 1981;142:601-4.

17 Campbell WB, Cole SEA, Skidmore R, Baird RM. The clinician and the vascular laboratory in the diagnosis of aortoiliac stenosis. Br F Surg 1984;71:302-6.

(Accepted 7 August 1986)

\title{
Medicine and the Media
}

\section{Public knowledge of AIDS and the DHSS advertisement campaign}

\author{
S MILLS, M J CAMPBELL, W E WATERS
}

A RECENT editorial claimed that the Department of Health and Social Security's advertising campaign to increase knowledge about the acquired immune deficiency syndrome (AIDS) had had little impact. ${ }^{1}$ The director of information at the DHSS, however, has called this claim subjective and has himself claimed that the advertisements provided useful new information about AIDS. ${ }^{2}$ We evaluated these claims by means of a questionnaire distributed before and after the campaign.

The first set of questionnaires about AIDS, together with a covering letter and a stamped addressed envelope, were posted in Southampton in February 1986, and similar questionnaires were posted in June 1986. The recipients of each questionnaire were chosen by a 1:500 systematic sample from the Southampton electoral roll, with a random, and different, starting name each time. In each case 300 questionnaires were posted, and the eventual response rates were $64 \%$ for the first survey and $66 \%$ for the second. In March and April the DHSS launched a publicity campaign, which appeared in all the major daily and Sunday newspapers. The

\footnotetext{
South Academic Block, Southampton General Hospital, Southampton SO9 4XY

S MILLS, medical student

M J CAMPBELL, PHD, lecturer in medical statistics

W E WATERS, FFCM, professor of community medicine

Correspondence to: Dr Campbell.
}

table compares the replies given before and after the campaign. Answers to question 1 were judged correct as long as the subject mentioned immune deficiency. In question 9 the ranges given were $0-99,100-499,500-999,1000-1999$, and 2000 or more cases (correct answer about 400). Questions 2-8, 10, and 11 were answered true, false, or don't know (questions 1-8 were answered explicitly by the DHSS advertisement). Answers were scored 1 for a correct answer, 0 for a don't know or missing answer, and -1 for an incorrect answer. The mean score before the advertising campaign was $5 \cdot 31$, and that afterwards was $5 \cdot 16$, the difference being $0.15(95 \%$ confidence interval $-0.30,0.60)$. The results thus indicate, if anything, a decrease in the level of public knowledge about AIDS. From the answers to individual questions there seems to be a slight increase in those who do not know what the initials AIDS stand for, and slightly fewer believe that AIDS is caused by a virus. In reply to the questions not covered by the DHSS advertisement more people answered don't know in the second survey when asked how many cases of AIDS there have been in this country, and more people in the second survey did not know whether women were at much less risk than men of catching AIDS.

In the second survey $31 \%$ claimed to have seen the DHSS advertisements. We also asked whether they had seen a poster campaign about AIDS run by Southampton City Council, which was in fact non-existent, and only $7 \cdot 5 \%$ admitted to having seen this. Of the 157 people who had not seen the council campaign, $31 \%$ had seen the DHSS advertisement. Of those who regularly read newspapers before the campaign, $46 \%$ (of 161) thought that the newspaper was a reliable source of information about AIDS, 